than the other $H e$ I lines. But the evidence is not sufficient to conclude that there is an appreciable effect of dilution. The absorption line $C$ II 4267 is strong.

The radial velocity obtained from ten strong $\mathrm{He}$ I lines and from $C a$ II 3933.66 is $+26 \mathrm{~km} / \mathrm{sec}$, whereas the central cores of the Balmer lines give $+47 \mathrm{~km} / \mathrm{sec}$. The difference of $+21 \mathrm{~km} / \mathrm{sec}$ is probably real.

The strongest $F e$ II lines are present as emissions superposed over broad absorptions. They are listed in Table I.

TABLE I

$F e$ il Lines in $\mu$ Centauri

$\begin{array}{cccc}\lambda \text { Lab. } & \text { Lab. Intensity } & \text { Notation } & \begin{array}{c}\text { Stellar } \\ \text { Intensity }\end{array} \\ 4233.17 & 11 & b^{4} P_{2 \frac{1}{2}}-z^{4} D^{0} & 2 \\ 4351.77 & 9 & b^{4} P_{1 \frac{1}{2}}-z^{4} D^{0} & 1 \\ 4555.89 & 8 & b^{4} F_{3 \frac{1}{2}}-z^{4} F^{0} & 1 \\ 4549.48 & 10 & b^{4} F_{3 \frac{1}{2}}-z^{4} D^{0} & 2 \\ 4583.84 & 11 & b^{4} F_{4 \frac{1}{2}}-z^{4} D^{0} & 2 \\ 4923.93 & 12 & a^{6} S_{2 \frac{1}{2}}-z^{6} P^{0} & 2 \\ 5018.45 & 12 & a^{6} S_{2 \frac{1}{2}}-z^{6} P^{0} & 2 \\ 5169.05 & 12 & a^{6} S_{2 \frac{1}{2}}-z^{6} P^{0^{2}}{ }_{3 \frac{1}{2}} & 3\end{array}$

McDonald Observatory April 1940

\title{
THE SPECTRUM OF T CORONAE BOREALIS
}

Bx P. Swings, C. T. Elvey, and O. Struve

T Coronae, which has shown an irregular increase in brightness since 1936, resembles $Z$ Andromedae, and probably consists of an M-type star and an early-type companion of nova-like character. ${ }^{1}$ Spectrograms of $\mathrm{T}$ Coronae were secured at the McDonald Observatory on the following dates: February 19, 20, and 21, April 20 and 24, 1940. The dispersions used were $53 \mathrm{~A} / \mathrm{mm}$ and $125 \mathrm{~A} / \mathrm{mm}$, at $\lambda 4000$. A strong continuous spectrum of early type is superposed over that of an $\mathrm{M}$ star of ap-

1 A. H. Joy, Pub. A.S.P., 50, 300, 1938 ; R. Minkowski, ibid., 51, 54, 1939 ; O. Hachenberg and P. Wellmann, Zs. f. Ap., 17, 246, 1939; P. Wellmann, ibid., 19, 16, 1939. 
proximately the same photographic magnitude. ${ }^{2}$ The following emission lines, which are all fairly sharp, were measured:

$$
\begin{aligned}
H & =H \alpha \text { to } \mathrm{H}_{21}, \text { and a strong Balmer continuum } \\
H e \mathrm{I} & =\lambda \lambda 3888.6(4), 3964.7(1), 4387.9(1), 4471.5(2), \\
& 4921.9(2), 5875.6(5) \\
H e \text { II }= & \lambda 4685.8(10) \\
{[O \text { II }] } & =\lambda \lambda 3726.2-3729.1(1) \\
C a \text { II }= & \lambda 3933.7(1) \\
{[O \text { III }]=} & N_{1}(2), N_{2}(1), \lambda 4363(0) \\
O \text { III }= & \lambda \lambda 3312.3(1), 3340.7(2), 3428.7(2), 3444.1(4), \\
& 3759.9(3) \\
N \text { III }= & \lambda \lambda 4097.3(2), 4634.2(1), 4640.6(1) \\
{[N e \text { III }] } & =\lambda 3868.7(2) \\
\text { Si } \mathrm{I} & =\lambda 3905.5(2)
\end{aligned}
$$

The $O$ III and $N$ III lines form incomplete multiplets which may be explained by Bowen's fluorescence mechanism. The radial velocities from the emission lines are : $H=-43 \mathrm{~km} / \mathrm{sec}$; $H e \mathrm{I}=-116 \mathrm{~km} / \mathrm{sec} ; H e$ II $=-75 \mathrm{~km} / \mathrm{sec} ; N$ III $=-78$ $\mathrm{km} / \mathrm{sec} ;[\mathrm{Ne} \mathrm{III}]=-11 \mathrm{~km} / \mathrm{sec}$. The difference in the radial velocities obtained from the $H$ and $H e$ I lines appears very strikingly in the case of the close pair $H 3889.05$ and $H e$ r 3888.65. On our plates the separation is much larger than $0.4 \mathrm{~A}$, and the two lines are not blended.

$\mathrm{H} \gamma$ and $\mathrm{H} \beta$ show absorption components of the P Cygni type, corresponding to a fairly large velocity of ejection (of the order of $300 \mathrm{~km} / \mathrm{sec}$ ). The degree of ionization has not increased much since 1938. The highest ionizations which we have observed correspond to the following ions:

$\mathrm{O}^{++}$(fluorescence and forbidden spectrum, ion. pot. of

$$
\mathrm{O}^{+}=34.9 \mathrm{v} \text {.) }
$$

$N^{++}$(fluorescence spectrum, I.P. of $N^{+}=29.4 \mathrm{v}$.)

$\mathrm{Ne}^{++}$(forbidden lines, I.P. of $\mathrm{Ne}^{+}=40.9 \mathrm{v}$.)

$\mathrm{He}^{+}$(recombination spectrum, I.P. of $\mathrm{He}^{+}=54.2$ v.)

There is no trace of $[\mathrm{Ne} \mathrm{v}]$ and $[\mathrm{Fe} \mathrm{VII}]$, which are outstanding features in AX Persei and CI Cygni. ${ }^{3}$

2 This is in agreement with the spectrophotometric measures by Wellmann (loc. cit.), based upon plates secured in June 1939.

${ }^{3} \mathrm{P}$. Swings and O. Struve, $A p$. $J$., in press. 
The relatively great intensity of the permitted incomplete multiplets of $O$ III is of interest. From the absence of $N_{1}, N_{2}$, and $\lambda 4363$ on their spectrograms, Hachenberg and Wellmann concluded that oxygen was mainly in the first stage of ionization. The present example illustrates the fact that under certain conditions of temperature and pressure, the ultraviolet fluorescence lines of $O$ III may be much more prominent than the forbidden lines.

McDonald Observatory
April 1940

NOTE ON HeI $\lambda \lambda 3965$ AND 5016

By J. A. HyneK

In the April issue of this journal Miss Davis ${ }^{1}$ reported that she observed $\lambda 5016$ to be sharp in $\zeta$ Tauri on two Coudé plates taken at the Mount Wilson Observatory. In view of previous notes on this line in this star and in $\varphi$ Persei by Biermann and Hachenberg ${ }^{2}$ and by myself, ${ }^{3}$ in which the line was reported to be diffuse (though Biermann found a sharp core on one plate), it may seem puzzling, especially to non-spectroscopic astronomers, that a matter as simple as the sharpness or diffuseness of a line should cause so much trouble observationally. I should like to report my recent observations of this line in $\varphi$ Persei and in $\zeta$ Tauri and to point out that the problem is not merely whether $\lambda 5016$ is sharp but whether $\lambda 5016$ and its companion line, $\lambda 3965$, which arises from the same metastable level, vary in appearance, and, if so, whether they vary alike. According to the theory of Struve and Wurm, ${ }^{4}$ both lines should, in the spectra of stars with extended envelopes such as $\varphi$ Persei and $\zeta$ Tauri, show a sharp core superposed on an extremely faint wide background line, provided that no additional mechanism operates. It was stated ${ }^{4}$ in support of the theory that $\lambda 3965$ in $\varphi$ Persei was always sharp, and, hence, presumably, $\lambda 5016$ also.

From a study of fifty Process spectrograms of $\varphi$ Persei, I
${ }_{1}$ Pub. A.S.P., 52, 147, 1940.
${ }^{2}$ Zs. f. Ap., 18, 89, 1939.
3 Ap. J., 89, 552, 1939.
${ }^{4}$ Ibid., 88, 84, 1938. 